\title{
Metastable vacua in superconformal SQCD-like theories
}

\author{
Antonio Amariti, ${ }^{a}$ Luciano Girardello, ${ }^{b}$ Alberto Mariotti, ${ }^{c}$ and Massimo Siani ${ }^{b}$ \\ ${ }^{a}$ Department of Physics, University of California \\ San Diego La Jolla, CA 92093-0354, U.S.A. \\ ${ }^{b}$ Dipartimento di Fisica, Università di Milano Bicocca and INFN, Sezione di Milano-Bicocca, \\ Piazza della Scienza 3, I-20126 Milano, Italy \\ ${ }^{c}$ Theoretische Natuurkunde, Vrije Universiteit Brussel and The International Solvay Institutes \\ Pleinlaan 2, B-1050 Brussels, Belgium \\ E-mail: antonio.amariti@physics.ucsd.edu, \\ luciano.girardello@mib.infn.it, alberto.mariotti@vub.ac.be, \\ massimo.siani@mib.infn.it
}

ABSTRACT: We study dynamical supersymmetry breaking in vector-like superconformal $\mathcal{N}=1$ gauge theories. We find appropriate deformations of the superpotential to overcome the problem of the instability of the non supersymmetric vacuum. The request for long lifetime translates into constraints on the physical couplings which in this regime can be controlled through efficient RG analysis.

KEYWORDS: Supersymmetry and Duality, Supersymmetry Breaking 


\section{Contents}

1 Introduction 1

2 The case of SQCD 3

3 Metastable vacua by adding relevant deformations $\quad 4$

$\begin{array}{lll}4 & \text { General strategy } & 11\end{array}$

5 Discussion $\quad 12$

$\begin{array}{ll}\text { A The renormalization of the bounce action } & 13\end{array}$

$\begin{array}{ll}\text { B The SSQCD } & 14\end{array}$

$\begin{array}{lll}\text { C RG flow and evolution of the scales } & 16\end{array}$

\section{Introduction}

In the last few years many models of metastable dynamical supersymmetry breaking (DSB) based on the ISS breakthrough [1] have been proposed (see [2] and references therein). Usually in DSB the strong dynamics jeopardizes the calculability of the model. The novelty of the approach of ISS relies in describing the low energy theory by the Seiberg dual phase $[3,4]$ which is weakly coupled in the IR. For a $\mathcal{N}=1 \mathrm{SU}\left(N_{c}\right)$ supersymmetric gauge theory with $N_{f}>N_{c}+1$ flavors the low energy physics can be equivalently described by a different magnetic $\mathrm{SU}\left(N_{f}-N_{c}\right)$ gauge group with $N_{f}$ flavors and a singlet.

The ISS model is based on SQCD with $N_{c}+1<N_{f}<3 / 2 N_{c}$ and small masses for the quarks. In this window the dual gauge theory at low energy flows to an IR free fixed point. This theory breaks supersymmetry at tree level in the small field region. In this region the strong dynamics effects are safely negligible and perturbation theory is reliable. The supersymmetric vacua are recovered in another region of the field space, namely at large vevs. The supersymmetry breaking vacuum is metastable and its lifetime can be made parametrically large by tuning the scales of the theory.

In principle the same mechanism is applicable in the conformal window if $N_{f} \gtrsim 3 / 2 N_{c}$, where there is a weakly interacting fixed point. In [1] the authors showed that in such window the non supersymmetric vacuum is unstable to decay because the strong dynamics effects are relevant and not negligible around the origin of the field space. Indeed the bounce action between the non supersymmetric vacuum and the supersymmetric one is not parametrically large, and the lifetime is short. Recent studies for realizing metastable vacua in the conformal window has been done in [5]. 
In this paper we deeply investigate this problem and we suggest viable model of metastable supersymmetry breaking in the conformal regime of a SQCD like theory.

We start our analysis by revisiting the ISS model in the conformal window, studying the RG evolution of the couplings and of the bounce action. The lifetime of the non supersymmetric vacuum is proportional to the ratio between the IR supersymmetry breaking scale and the IR holomorphic scale as expected. We find that this ratio depends only on the gauge coupling calculated at the conformal fixed point. This shows that the lifetime of the vacuum cannot be parametrically large below the IR scale at which the theory exits from the conformal regime.

Nevertheless, we argue that by adding some deformations the metastable vacua can still exist in the conformal window. We propose a deformation of the ISS model, by adding a small number of massive quarks and some new singlets in the dual description of massive SQCD. This model is a $\mathrm{SU}(N)$ SCFT dual to the SSQCD defined in [6] with some relevant deformations. When these deformations are small, the theory is approximately a CFT. In such approximate CFT regime this theory is interacting, and we restrict to $N_{f} \gtrsim 3 / 2 N_{c}$, where a reliable perturbative analysis is sensible. This model can evade the argument of ISS because the new massive fields modify the non perturbative superpotential and thus the supersymmetric vacuum. As a consequence the bounce action has a parametrical behavior in terms of the relevant deformations. The lifetime can be large if we impose some constraints on the physical couplings at the CFT exit scale.

Differently from the IR free case, in which the low energy theory is free, in this case it is interacting. The anomalous dimensions of the fields are not zero, and the Kahler potential is renormalized. This implies that the physical couplings undergo RG evolution in the approximate CFT regime. The constraints for the stability of the non supersymmetric vacuum have to be imposed on the physical IR couplings after the RG evolution.

The paper is organized as follows. In section 2 we discuss the obstructions to the existence of metastable vacua in the conformal window of SQCD, and we introduce the analysis of the RG evolution for the couplings and the holomorphic scale. In the main section 3 we outline our strategy for the search of metastable vacua by studying the SSQCD model appropriately deformed. The essential idea just relies on the features of super CFT, where RG analysis and determination of anomalous dimensions are feasible. In section 4 we discuss the generalization of our analysis to $\mathcal{N}=1 \mathrm{SCFT}$ s and give a general procedure to generate new models of conformal supersymmetry breaking. In section 5 we conclude and comment on possible applications. In the appendix A we study the RG flow associated with the bounce action. In the appendix B we review the Seiberg duality in SSQCD and discuss the origin of the relevant couplings.

While we were completing this paper, the work [7] appeared which has some overlap with our results. 


\section{The case of SQCD}

In their original paper [1] the authors studied a $\mathrm{SU}\left(N_{c}\right)$ gauge theory with $N_{f}$ flavors of quarks charged under an $\mathrm{SU}\left(N_{f}\right)^{2}$ flavor symmetry broken to $\mathrm{SU}\left(N_{f}\right)$ by the superpotential

$$
W=m Q \tilde{Q}
$$

where the mass $m$ is much smaller than the holomorphic scale of the theory $\Lambda$. In the window $N_{c}+1<N_{f}$ this theory admits a dual description in term of a magnetic gauge group $\mathrm{SU}(\tilde{N})=\mathrm{SU}\left(N_{f}-N_{c}\right), N_{f}$ magnetic quarks $q$ and $\tilde{q}$ and the electric meson $N=Q \tilde{Q}$ normalized to have mass dimension one. The dual superpotential reads

$$
W_{m}=-h \mu^{2} N+h N q \tilde{q}+\tilde{N}\left(\tilde{\Lambda}^{\tilde{b}} h^{N_{f}} \operatorname{det} N\right)^{\frac{1}{\tilde{N}}}
$$

where we introduced the marginal coupling $h$ and the holomorphic scale of the dual theory $\tilde{\Lambda}$, and we added the non perturbative contribution due to gaugino condensation. From now on we set $h=1$. The holomorphic scales $\Lambda$ and $\tilde{\Lambda}$ are related by a scale matching relation [4]. The one loop beta function coefficient is $\tilde{b}=3 \tilde{N}-N_{f}=2 N_{f}-3 N_{c}$.

In the range $N_{c}+1<N_{f}<3 / 2 N_{c}$, this theory has a supersymmetry breaking vacuum at $N=0$, with non zero vev for the quarks. The supersymmetric vacuum is recovered in the large field region for $N$. The parametrically long distance between the two vacua guarantees the long life time of the non supersymmetric one.

The metastable non supersymmetric vacua found in the magnetic free window of massive SQCD are destabilized in the conformal window $3 / 2 N_{C}<N_{f}<3 N_{C}$. This fact is based on the observation that the non perturbative superpotential in (2.2) is not negligible in the small field region, as instead it happens in the magnetic free window.

Here we give a pedagogical derivation of the behavior of the SQCD bounce action in the conformal window. As stated in [1], while in the free magnetic phase there are two parameters, $m$ and $\Lambda$, in the conformal phase $\Lambda$ is not a parameter anymore. Thus, there is no possibility to get a dimensionless parameter to guarantee an arbitrary large lifetime for the metastable state. We show this intuitive idea with an explicit computation which will be useful in the rest of our analysis. In general, in the presence of relevant deformations the conformal regime is only approximated. If these deformation are small enough there is a large regime of scales in which the theory flows to lower energies while remaining approximatively conformal. The physical couplings vary along the RG flow because of the wave function renormalization of the fields, until the theory exits from the conformal regime. Below this scale the theory is IR free and the renormalization effects are negligible.

We study the RG properties of the ISS model in the conformal window by using a canonical basis for the fields. Flowing from a UV scale $E_{\mathrm{UV}}$ to an IR scale $E_{\mathrm{IR}}$ the fields are not canonically normalized anymore, and we have to renormalize them by the wave function renormalization $Z_{i}\left(E_{\mathrm{IR}}, E_{\mathrm{UV}}\right)$, namely $\phi_{i}^{\mathrm{IR}}=\sqrt{Z}_{i} \phi_{i}^{\mathrm{UV}}$. In terms of the renormalized fields the Kahler potential is canonical. The couplings appearing in the superpotential undergo RG evolution, and are the physical couplings. In this way the coupling $\mu_{\mathrm{IR}}$ of the IR superpotential becomes

$$
\mu_{\mathrm{IR}}=\mu_{\mathrm{UV}} Z_{N}\left(E_{\mathrm{IR}}, E_{\mathrm{UV}}\right)^{-\frac{1}{4}}
$$


The holomorphic scale that appears in the superpotential is unphysical in the conformal window and it is defined as

$$
\tilde{\Lambda}=E e^{-\frac{8 \pi^{2}}{g_{*}^{2} \tilde{b}}}
$$

where $\mathrm{E}$ is the RG running scale, and $g_{*}$ is the gauge coupling at the superconformal fixed point. In the canonical basis $\tilde{\Lambda}$ is rescaled as well during the RG conformal evolution as $[8-10]$

$$
\tilde{\Lambda}_{\mathrm{IR}}=\tilde{\Lambda}_{\mathrm{UV}} \frac{E_{\mathrm{IR}}}{E_{\mathrm{UV}}}
$$

In the ISS model the two possible sources of breaking of the conformal invariance are the masses of the fields at the non supersymmetric vacuum and at the supersymmetric vacuum. We define the CFT exit scale as $E_{\mathrm{IR}}=\Lambda_{c}$. In this model this scale is necessarily set by the masses of the fields at the supersymmetric vacuum, which are proportional to the vev of the field $N$. In fact by setting

$$
\Lambda_{c} \equiv\langle N\rangle_{\text {susy }}=\mu_{\mathrm{IR}}\left(\frac{\mu_{\mathrm{IR}}}{\tilde{\Lambda}_{\mathrm{IR}}}\right)^{\frac{\tilde{b}}{N_{f}-\tilde{N}}}
$$

the physical mass at this scale results

$$
\mu_{\mathrm{IR}}=\Lambda_{c} e^{-\frac{4 \pi^{2}}{g *^{2} N}} \ll \Lambda_{c}
$$

Hence the assumption that $\langle N\rangle_{\text {susy }}$ stops the conformal regime is consistent. The opposite case, with $\Lambda_{c} \equiv \mu_{\mathrm{IR}} \gg\langle N\rangle_{\text {susy }}$ cannot be consistently realized.

The bounce action at the scale $\Lambda_{c}$ is

$$
S_{B} \sim\left(\frac{\mu_{\mathrm{IR}}}{\tilde{\Lambda}_{\mathrm{IR}}}\right)^{\frac{4 \tilde{b}}{N_{f}-\tilde{N}}} \sim e^{\frac{16 \pi^{2}}{g_{*}^{2} \tilde{N}}}
$$

This bounce is not parametrically large and it depends only on the coupling constant $g_{*}$ at the fixed point. In general, as we shall see in the appendix A, the bounce action is not RG invariant, but it runs during the RG flow. In this case $S_{B}$ at the CFT exit scale only depends on the ratio of the two relevant scales in the theory which is the RG invariant coupling constant.

In general, by adding other deformations, the bounce action is not RG invariant anymore and we have to study its flow. In some cases, the lifetime of a vacuum decreases as we flow towards the infrared. In the next section, by adding new massive quarks to the ISS model, we show that long living metastable vacua exist in the conformal window.

\section{Metastable vacua by adding relevant deformations}

In this section we describe our proposal for realizing metastable supersymmetry breaking in the conformal window of $N=1$ SQCD-like theories. The key point is the addition of massive quarks in the dual magnetic description. This introduces a new mass scale that controls the distance in the field space of the supersymmetric vacua. 


\begin{tabular}{|c||c|c|c|}
\hline & $N_{f}^{(1)}$ & $N_{f}^{(2)}$ & $\tilde{N}$ \\
\hline$N$ & $N_{f}^{(1)} \otimes N_{f}^{(1)}$ & 1 & 1 \\
$q+\tilde{q}$ & $\bar{N}_{f}^{(1)} \oplus N_{f}^{(1)}$ & 1 & $\tilde{N} \oplus \overline{\tilde{N}}$ \\
$p+\tilde{p}$ & 1 & $\bar{N}_{f}^{(2)} \oplus N_{f}^{(2)}$ & $\tilde{N} \oplus \overline{\tilde{N}}$ \\
$K+L$ & $\bar{N}_{f}^{(1)} \oplus N_{f}^{(1)}$ & $N_{f}^{(2)} \oplus \bar{N}_{f}^{(2)}$ & 1 \\
\hline
\end{tabular}

Table 1. Matter content of the dual SSQCD.

We start from the magnetic description of the ISS model of the previous section. We add a new set of massive fields $p$ and $\tilde{p}$ charged under a new $\mathrm{SU}\left(N_{f}^{(2)}\right)$ flavor symmetry. We also add new bifundamental fields $K$ and $L$ charged under $\mathrm{SU}\left(N_{f}^{(1)}\right) \times \mathrm{SU}\left(N_{f}^{(2)}\right)$. The added number of flavors is such that $3 / 2 \tilde{N}<N_{f}^{(1)}+N_{f}^{(2)}<3 \tilde{N}$. The superpotential of the model is

$$
W=K p \tilde{q}+L \tilde{p} q+N q \tilde{q}+\rho p \tilde{p}-\mu^{2} N
$$

and the field content is summarized in the table 1. This model corresponds to the dual description of the SSQCD studied in [6], deformed by two relevant operators. In the appendix B we show the Seiberg dual electric description of this theory, and we discuss a mechanism to dynamically generate the mass term for the new quarks.

In the rest of this section we shall show that in the case $N_{f}^{(1)}>\tilde{N}$ there are ISS like metastable supersymmetry breaking vacua if we are near the IR free border of the conformal window

$$
3 \tilde{N} \gtrsim N_{f}^{(1)}+N_{f}^{(2)}
$$

The non supersymmetric vacuum. The non supersymmetric vacuum is located near the origin of the field space where the superpotential (3.1) can be studied perturbatively. Appropriate bounds on the parameters $\rho$ and $\mu$ allow to neglect the non perturbative dynamics. We will see that these bounds can be consistent with the running of the coupling constants.

Tree level supersymmetry breaking is possible if

$$
N_{f}^{(1)}>\tilde{N} \quad \Rightarrow \quad 2 \tilde{N}>N_{f}^{(2)}
$$

where the second inequality follows from (3.2). The equation of motion for the field $N$ breaks supersymmetry through the rank condition mechanism. We solve the other equations of motion and we find the non supersymmetric vacuum

$$
\begin{array}{lll}
q=\left(\begin{array}{c}
\mu+\sigma_{1} \\
\phi_{1}
\end{array}\right) & \tilde{q}=\left(\mu+\sigma_{2} \phi_{2}\right) & N=\left(\begin{array}{cc}
\sigma_{3} & \phi_{3} \\
\phi_{4} & X
\end{array}\right) \\
p=\phi_{5} & \tilde{p}=\phi_{6} & L=\left(\begin{array}{ll}
\phi_{7} & \tilde{Y}
\end{array}\right) \quad K=\left(\begin{array}{c}
\phi_{8} \\
Y
\end{array}\right)
\end{array}
$$


where we have also inserted the fluctuations around the minimum, $\sigma_{i}$ and $\phi_{i}$. The fields $X, Y$ and $\tilde{Y}$ are pseudomoduli. The infrared superpotential is

$$
\begin{aligned}
W_{\mathrm{IR}}= & X \phi_{1} \phi_{2}-\mu^{2} X+\mu\left(\phi_{1} \phi_{4}+\phi_{2} \phi_{3}\right)+\mu\left(\phi_{5} \phi_{8}+\phi_{6} \phi_{7}\right) \\
& +Y \phi_{2} \phi_{5}+\tilde{Y} \phi_{1} \phi_{6}+\rho \phi_{5} \phi_{6}
\end{aligned}
$$

In the limit of small $\rho$, this is the same superpotential studied in [11]. This superpotential corresponds to the one studied in [12] in the $R$ symmetric limit. The fields $X, Y$ and $\tilde{Y}$ are stabilized by one loop corrections at the origin with positive squared masses.

The supersymmetric vacuum. We derive here the low energy effective action for the field $N$, and we recover the supersymmetric vacuum in the large field region. The supersymmetric vacuum is characterized by a large expectation value for $N$. This vev gives mass to the quarks $q$ and $\tilde{q}$ and we can integrate them out at zero vev. Also the quarks $p$ and $\tilde{p}$ are massive and are integrated out at low energy. The scale of the low energy theory $\Lambda_{L}$ is related to the holomorphic scale $\tilde{\Lambda}$ via the scale matching relation

$$
\Lambda_{L}^{3 \tilde{N}}=\tilde{\Lambda}^{3 \tilde{N}-N_{f}^{(1)}-N_{f}^{(2)}} \operatorname{det} \rho \operatorname{det} N
$$

The resulting low energy theory is $\mathcal{N}=1 \mathrm{SYM}$ plus a singlet, with effective superpotential

$$
W=-\mu^{2} N+\tilde{N}\left(\tilde{\Lambda}^{3 \tilde{N}-N_{f}^{(1)}-N_{f}^{(2)}} \operatorname{det} \rho \operatorname{det} N\right)^{1 / \tilde{N}}
$$

where the last term is the gaugino condensate. By solving the equation of motion for $N$ we find the supersymmetric vacuum

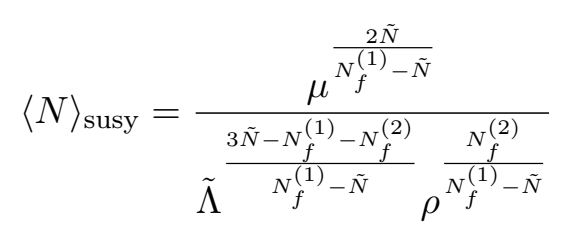

Lifetime. The lifetime of the non supersymmetric vacuum is controlled by the bounce action to the supersymmetric vacuum. In this case, the triangular approximation [13] is valid and the action can be approximated as $S_{B} \simeq(\Delta \Phi)^{4} /(\Delta V)$. If we estimate $\Delta \Phi \sim$ $\langle N\rangle_{\text {susy }}$ and $\Delta V \sim \mu^{4}$ we obtain

$$
S_{B}=\left(\frac{\tilde{\Lambda}}{\rho}\right)^{\frac{4 N_{f}^{(2)}}{N_{f}^{(1)}-\tilde{N}}}\left(\frac{\mu}{\tilde{\Lambda}}\right)^{\frac{12 \tilde{N}-4 N_{f}^{(1)}}{N_{f}^{(1)}-\tilde{N}}}
$$

This expression is not automatically very large since $\mu \ll \tilde{\Lambda}$. However, we can impose the following bound on $\rho$

$$
\langle N\rangle_{\text {susy }} \gg \mu \quad \rightarrow \quad \rho \ll \tilde{\Lambda}\left(\frac{\mu}{\tilde{\Lambda}}\right)^{\left(3 \tilde{N}-N_{f}^{(1)}\right) / N_{f}^{(2)}}
$$

If this bound is satisfied, the supersymmetric and the non supersymmetric vacua are far away apart in the field space and the non perturbative terms can be neglected at the 
supersymmetry breaking scale. This differs from the ISS model in the conformal window. In that case the non-perturbative effects became important at the supersymmetry breaking scale. The bounce action was proportional to the gauge coupling constant at the fixed point and it was impossible to make it parametrically long. The introduction of the new mass scale $\rho$ allows a solution to this problem.

The bound (3.10) should be imposed on the IR couplings at the CFT exit scale $E_{\mathrm{IR}}=$ $\Lambda_{c}$. In this case we have a new possible source of CFT breaking, namely the relevant deformation $\rho$. However we look for a regime of couplings such that the CFT exit scale is set by the supersymmetric vacuum scale, i.e. $\Lambda_{c}=\langle N\rangle_{\text {susy }} \gg \mu_{\mathrm{IR}}, \rho_{\mathrm{IR}}$. The scale $\Lambda_{c}$ is

$$
\Lambda_{c}=\langle N\rangle_{\text {susy }}=\tilde{\Lambda}_{\mathrm{IR}}\left(\frac{\mu_{\mathrm{IR}}}{\tilde{\Lambda}_{\mathrm{IR}}}\right)^{\frac{2 \tilde{N}}{N_{f}^{(1)}-\tilde{N}}}\left(\frac{\tilde{\Lambda}_{\mathrm{IR}}}{\rho_{\mathrm{IR}}}\right)^{\frac{N_{f}^{(2)}}{N_{f}^{(1)}-\tilde{N}}}
$$

At this scale we define $\epsilon_{\mathrm{IR}}$ as the ratio between the IR masses $\rho_{\mathrm{IR}}$ and $\mu_{\mathrm{IR}}$ and we demand that

$$
\epsilon_{\mathrm{IR}}=\frac{\rho_{\mathrm{IR}}}{\mu_{\mathrm{IR}}} \ll 1
$$

Rearranging (3.11) for $\mu_{\mathrm{IR}}$ and $\rho_{\mathrm{IR}}$ we have

$$
\begin{gathered}
\mu_{\mathrm{IR}}=\Lambda_{c} e^{-\frac{8 \pi^{2}}{g_{*}^{2}\left(2 \tilde{N}-N_{f}^{(2)}\right)}} \epsilon_{\mathrm{IR}}^{\frac{N_{f}^{(2)}}{2 \tilde{N}-N_{f}^{(2)}}} \ll \Lambda_{c} \\
\rho_{\mathrm{IR}}=\Lambda_{c} e^{-\frac{8 \pi^{2}}{g_{*}^{2}\left(2 \tilde{N}-N_{f}^{(2)}\right)}} \epsilon_{\mathrm{IR}}^{\frac{2 \tilde{N}}{2 \tilde{N} N_{f}^{(2)}}} \ll \Lambda_{c}
\end{gathered}
$$

This shows that requiring $\epsilon_{\mathrm{IR}} \ll 1$ is consistent with the CFT exit scale to be $\langle N\rangle_{\text {susy }}$.

By substituting (3.11) and (3.13) in (3.9), the bounce action becomes

$$
S_{B}=\frac{e^{\frac{32 \pi^{2}}{g_{*}^{2}\left(2 \tilde{N}-N_{f}^{(2)}\right)}}}{\frac{4 N_{f}^{(2)}}{2 \tilde{N}-N_{f}^{(2)}}}
$$

and in the limit $N_{f}^{(2)} \rightarrow 0$ it reduces to the one computed in the (2.8). Here the bounce is not only proportional to a numerical factor depending on $g_{*}^{2}$, but there is also a parameter, relating the ratios of the physical masses $\rho_{\mathrm{IR}}$ and $\mu_{\mathrm{IR}}$ at the CFT exit scale. The bounce action can be large if $\epsilon_{\mathrm{IR}} \ll 1$, providing a parametrically large lifetime for the non supersymmetric vacuum.

Using the RG evolution equations the bound $\epsilon_{\mathrm{IR}} \ll 1$ translates in constraints on the UV masses $\rho_{\mathrm{UV}}$ and $\mu_{\mathrm{UV}}$ at the UV scale. These masses are relevant perturbations and their ratio must be small along the RG flow. 
RG flow in the approximate conformal regime. The relevant coupling constants run from $E_{\mathrm{UV}}$ to $E_{\mathrm{IR}}=\Lambda_{c}$. We require that these terms are so small in the UV to be considered as perturbations of the CFT, i.e. $\rho_{\mathrm{UV}}, \mu_{\mathrm{UV}} \ll \Lambda_{\mathrm{UV}}$.

The ratio $\epsilon_{\mathrm{UV}}$ given at the scale $E_{\mathrm{UV}}$ runs as the coupling constants down to $\Lambda_{c}$. We now study the evolution of this ratio. The requirement of long lifetime of the metastable vacuum (3.10) corresponds to $\epsilon_{\mathrm{IR}} \ll 1$ and it constrains both $\epsilon_{\mathrm{UV}}$ and the duration of the approximate conformal regime, $\Lambda_{c} / E_{\mathrm{UV}}$.

The running of the relevant couplings in the conformal windows is parameterized by the equations

$$
\begin{aligned}
& \rho_{\mathrm{IR}}=\rho_{\mathrm{UV}} Z_{p}\left(\Lambda_{c}, E_{\mathrm{UV}}\right)^{-1 / 2} Z_{\tilde{p}}\left(\Lambda_{c}, E_{\mathrm{UV}}\right)^{-1 / 2} \\
& \mu_{\mathrm{IR}}=\mu_{\mathrm{UV}} Z_{N}\left(\Lambda_{c}, E_{\mathrm{UV}}\right)^{-1 / 4}
\end{aligned}
$$

The wave function renormalization $Z$ is obtained by integrating the equation

$$
\frac{\mathrm{d} \log Z_{i}}{\mathrm{~d} \log E}=-\gamma_{i}
$$

from $E_{\mathrm{UV}}$ to $\Lambda_{c}$, where $\gamma_{i}$ is constant in the conformal regime, and it reads

$$
Z_{\phi}\left(\Lambda_{c}, E_{\mathrm{UV}}\right)=\left(\frac{\Lambda_{c}}{E_{\mathrm{UV}}}\right)^{-\gamma_{\phi_{i}}}
$$

The physical couplings at the CFT exit scale are

$$
\rho_{\mathrm{IR}}=\rho_{\mathrm{UV}}\left(\frac{\Lambda_{c}}{E_{\mathrm{UV}}}\right)^{\gamma_{p}}, \quad \mu_{\mathrm{IR}}=\mu_{\mathrm{UV}}\left(\frac{\Lambda_{c}}{E_{\mathrm{UV}}}\right)^{\gamma_{N} / 4}
$$

where we have used the relation $\gamma_{p}=\gamma_{\tilde{p}}$.

The ratio $\epsilon$ evolves as

$$
\epsilon_{\mathrm{IR}}=\epsilon_{\mathrm{UV}}\left(\frac{\Lambda_{c}}{E_{\mathrm{UV}}}\right)^{\gamma_{p}-\gamma_{N} / 4}
$$

and we demand that it is $\epsilon_{\mathrm{IR}} \ll 1$ in order to satisfy the stability constraint for the non supersymmetric vacuum. The flow from $\epsilon_{\mathrm{UV}}$ to $\epsilon_{\mathrm{IR}}$ depends on $\Lambda_{c} / E_{\mathrm{UV}}$ and on the anomalous dimensions. The precise relation between $\epsilon_{\mathrm{UV}}$ and $\epsilon_{\mathrm{IR}}$ is found by calculating the exact value of $\gamma_{p}$ and $\gamma_{N}$. The anomalous dimensions of the fields $\phi_{i}$ are obtained from the relation $\Delta_{i}=1+\gamma_{i} / 2$ where $\Delta_{i}=\frac{3}{2} R_{i}$. The $R$ charges can be computed by using a-maximization.

The a-maximization procedure, defined in [14], shows that in SCFT the correct $R$ charge at the fixed point is found by maximizing the function

$$
a_{\text {trial }}(R)=\frac{3}{32}\left(3 \operatorname{Tr} R^{3}-\operatorname{Tr} R\right)
$$

The $R$-charges in (3.21) are all the non anomalous combinations of the $R_{0}$ charges under which the supersymmetry generators have charge -1 and all the other flavor symmetries commuting with the supersymmetry generators. The $\operatorname{Tr}\left(R^{3}\right)$ and $\operatorname{Tr}(R)$ are the coefficients 
of the gauge anomaly and gravitational anomaly. The $R$-charges that maximize (3.21) are the $R$ charges appearing in the superconformal algebra.

The $R$ charge assignment has to satisfy the anomaly free condition and the constraint that the superpotential couplings should be marginal. These conditions are

$$
\tilde{N}+N_{f}^{(1)}(R[q]-1)+N_{f}^{(2)}(R[p]-1)=0, \quad R[p]+R[q]+R[L]=2, \quad R[N]+2 R[q]=2
$$

where the symmetry enforces $R[q]=R[\tilde{q}], R[p]=R[\tilde{p}]$ and $R[K]=R[L]$. The $a_{\text {trial }}$ function that has to be maximized is

$$
\begin{aligned}
a_{\text {trial }}= & \frac{3}{32}\left(2 N_{f}^{(1)} \tilde{N}\left(3(R[q]-1)^{3}-R[q]+1\right)+2 N_{f}^{(2)} \tilde{N}\left(3(R[p]-1)^{3}-R[q]+1\right)\right. \\
& \left.+2 N_{f}^{(1)} N_{f}^{(2)}\left(3(R[L]-1)^{3}-R[L]+1\right)+N_{f}^{(1) 2}\left(3(R[N]-1)^{3}-R[N]+1\right)+2 \tilde{N}^{2}\right)
\end{aligned}
$$

By defining $R[N]=2 y$ we have $R[q]=1-y$. The other $R$ charges are

$$
R[p]=\frac{1}{n}(n-x+y), \quad R[L]=y+\frac{x-y}{n}
$$

where $n=\frac{N_{f}^{(2)}}{N_{f}^{(1)}}$ and $x=\frac{\tilde{N}}{N_{f}^{(1)}}$. We can simplify the a maximization in terms of the only variable $y$, obtaining

$$
y_{\max }=\frac{-3\left(n+n^{3}\right)+3(-1+n)^{2} x-3 x^{2}+\sqrt{n^{2}\left(n^{4}-8 n(x-1)+8 n^{3}(x-1)+9(x-1)^{4}-6 n^{2}(1+3(x-2) x)\right)}}{3\left(1-n\left(3+n+n^{2}\right)+\left(-1+n^{2}\right) x\right)}
$$

Once we know the anomalous dimensions and once we fix the duration of the approximate conformal regime we can see what is the bound to impose on the UV ratio $\epsilon_{\mathrm{UV}}=\rho_{\mathrm{UV}} / \mu_{\mathrm{UV}}$ such that

$$
\epsilon_{\mathrm{IR}}=\epsilon_{\mathrm{UV}}\left(\frac{\Lambda_{c}}{E_{\mathrm{UV}}}\right)^{\frac{3}{2 n}(n-2 x+2 y-y n)} \ll 1
$$

As a result of the a-maximization procedure, we find that $\gamma_{N}>0$ and $\gamma_{p}<0$ in the range (3.2). The former is a consequence of the unitarity of the theory. Thus, from (3.19) we note that the coupling $\mu$ is suppressed during the RG flow, while $\rho$ is enhanced, and (3.12) poses a nontrivial constraint on the coupling constants of the theory at the CFT exit scale.

In the figures $1-6$ we have plotted some region of the ranks $x$ and $n$ by fixing $\epsilon_{\mathrm{UV}}$ and $\Lambda_{c} / E_{\mathrm{UV}}$. The colored parts of the figures correspond to $\epsilon_{\mathrm{IR}}<1$. We represented the behavior of $\epsilon_{\mathrm{IR}}$ varying from zero to one by changing the color of the shaded region from red to orange and then to yellow. The white part of the figures represents the region in which $\epsilon_{\mathrm{IR}}>1$. We also marked the border line between the magnetic IR free and the conformal window, corresponding to $3 \tilde{N}=\left(N_{f}^{(1)}+N_{f}^{(2)}\right)$.

From the figures we see that smaller values of the ratio $\epsilon_{\mathrm{UV}}$ guarantee that the running can be longer in the CFT window. The red region shaded in the figures, near $N_{f}^{(1)}+N_{f}^{(2)}=$ $3 \tilde{N}$, is filled also if the running is extended over a large regime of scales. At the lower edge 


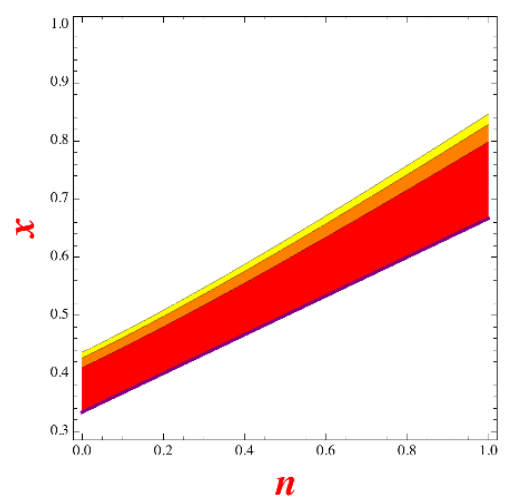

Figure 1. $\frac{\rho_{\mathrm{UV}}}{\mu_{\mathrm{UV}}}=10^{-2}, \frac{\Lambda_{c}}{E_{\mathrm{UV}}}=10^{-4}$

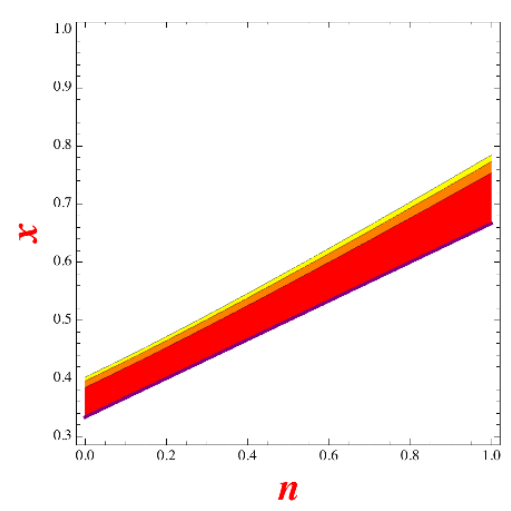

Figure 3. $\frac{\rho_{\mathrm{UV}}}{\mu_{\mathrm{UV}}}=10^{-2}, \frac{\Lambda_{c}}{E_{\mathrm{UV}}}=10^{-6}$

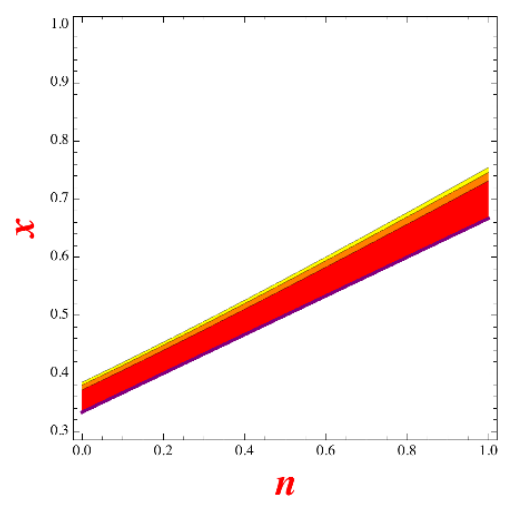

Figure 5. $\frac{\rho_{\mathrm{UV}}}{\mu_{\mathrm{UV}}}=10^{-2}, \frac{\Lambda_{c}}{E_{\mathrm{UV}}}=10^{-8}$

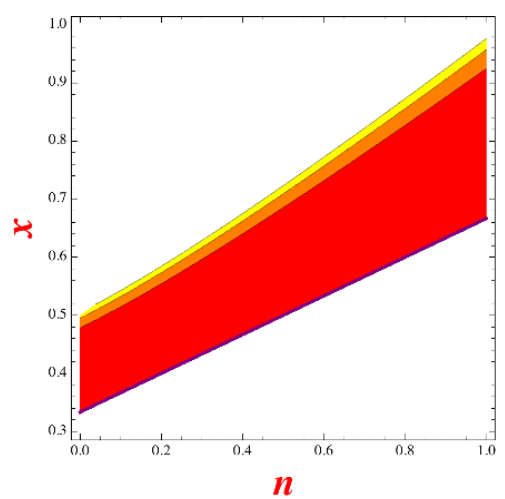

Figure 2. $\frac{\rho_{\mathrm{UV}}}{\mu_{\mathrm{UV}}}=10^{-4}, \frac{\Lambda_{c}}{E_{\mathrm{UV}}}=10^{-4}$

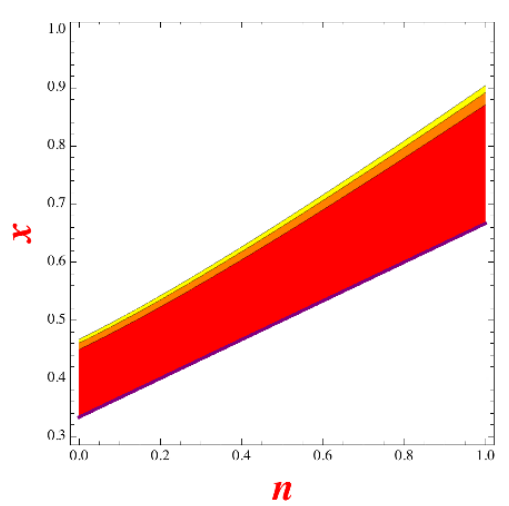

Figure 4. $\frac{\rho_{\mathrm{UV}}}{\mu_{\mathrm{UV}}}=10^{-4}, \frac{\Lambda_{c}}{E_{\mathrm{UV}}}=10^{-6}$

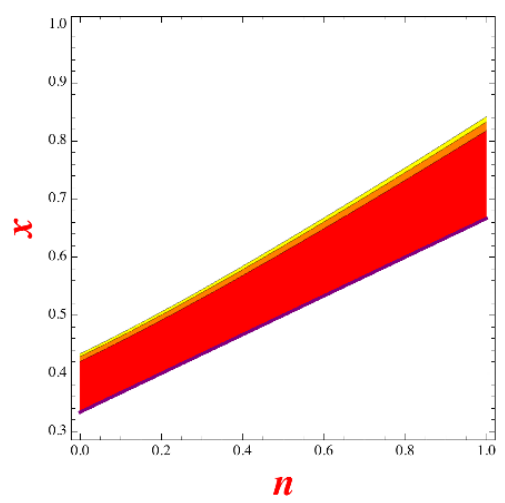

Figure 6. $\frac{\rho_{\mathrm{UV}}}{\mu_{\mathrm{UV}}}=10^{-4}, \frac{\Lambda_{c}}{E_{\mathrm{UV}}}=10^{-8}$

of this region the anomalous dimensions are close to zero, the UV hierarchy imposed on the relevant deformations is preserved during the flow, and $\epsilon_{\mathrm{UV}} \sim \epsilon_{\mathrm{IR}}$.

Very close to the $3 \tilde{N}=\left(N_{f}^{(1)}+N_{f}^{(2)}\right)$ line, our model represents a calculable metastable supersymmetry breaking theory. Indeed in this region the perturbative analysis suffices, 
and from the figures $1-6$ the lifetime is parametrically large. As we move away from the $3 \tilde{N}=\left(N_{f}^{(1)}+N_{f}^{(2)}\right)$ line the anomalous dimensions get larger. Even if the shaded region spreads far from this line, we cannot rely on the perturbative computations we made above. Thus, in this case the non perturbative effects get larger and a deeper analysis is necessary.

In conclusion we have found regions in the parameter space where the theory possesses metastable non supersymmetric vacua. The RG flow analysis gives non trivial constraints on the relevant deformations and on the duration of the approximate conformal regime.

\section{General strategy}

We discuss here the generalization of the mechanism of supersymmetry breaking in SCFTs deformed by relevant operators. As in SSQCD, the lifetime of the metastable vacuum can be long in the conformal window of other models, with opportune choices of the parameters.

Consider a SU $\left(N_{c}\right)$ gauge theory with $N_{f}^{(1)}$ flavors of quarks in the magnetic IR free window and with a metastable supersymmetry breaking vacuum in the dual phase. In the magnetic phase a new set of $N_{f}^{(2)}$ massive quarks must be added to reach the conformal window. If there is some gauge invariant operator $\mathcal{O}$ that hits the unitary bounds, $R(\mathcal{O})<2 / 3$, it is necessary to add other singlets and also marginal couplings in the superpotential between the quarks and these new singlets. The mass term for the new quarks is a relevant perturbation which grows in the infrared, and it has to be very small with respect to the other scales of the theory, down to the CFT exit scale. This mass term modifies the non perturbative superpotential and the supersymmetric vacuum, which sets the CFT exit scale. One must inspect a regime of couplings such that the supersymmetric vacuum is far away in the field space. This regime corresponds to a bound on the parameters of the theory, which have to be consistent with the RG running of the physical coupling constants. In the canonical basis the running of the physical couplings can be absorbed in the superpotential by the wave function renormalization of the fields. If there is a relevant operator $\Delta W=\eta \mathcal{O}$, with classical dimension $\operatorname{dim}(\mathcal{O})=d$, the physical coupling $\eta$ runs from the UV scale $E_{\mathrm{UV}}$ to the IR scale $E_{\mathrm{IR}}$ as

$$
\eta\left(E_{\mathrm{IR}}\right)=\eta\left(E_{\mathrm{UV}}\right) Z_{\mathcal{O}}\left(E_{\mathrm{IR}}, E_{\mathrm{UV}}\right)^{-\frac{1}{2}}=\eta\left(E_{\mathrm{UV}}\right)\left(\frac{E_{\mathrm{IR}}}{E_{\mathrm{UV}}}\right)^{\gamma / 2}
$$

We require that the running in this approximate conformal regime stops at the energy scale $\Lambda_{c}$ set by the masses at the supersymmetric vacuum. The bounds on the parameters that ensure the stability of the metastable vacuum have to hold at this IR CFT exit scale. The equation (4.1) translates these bounds in some requirements on the UV deformations. The metastable vacua have long lifetime if there is some regime of UV couplings in which the stability requirements are satisfied in the weakly coupled conformal window.

Here we have shown that in SSQCD there are some regions in the conformal window in which a large hierarchy among the couplings allows the existence of long living metastable vacua. We expect other models with this behavior. 


\section{Discussion}

In this paper we discussed the realization of the ISS mechanism in the conformal window of SQCD-like theory. In [1] the metastable vacua disappeared if $3 / 2 N_{c}<N_{f}<3 N_{c}$ because the non perturbative dynamics was not negligible in the small field region, and this destabilized the non supersymmetric vacua.

We have reformulated this problem in terms of the RG flow from the $U V$ cut-off of the theory down to the CFT exit scale. In the ISS model the CFT exit scale and the supersymmetry breaking scale are proportional because of the equation of motion of the meson. Their ratio depends only on the gauge coupling constant at the fixed point. The bounce action is proportional to this ratio and cannot be parametrically long.

This behavior suggests a mechanism to evade the problem and to build models with long living metastable vacua in the conformal window of SQCD-like theories. A richer structure of relevant deformations than in the ISS model is necessary. Metastable vacua with a long lifetime can exist if the bounce action at the CFT exit scale depends on the relevant deformations and it is not RG invariant. We have studied this mechanism in an explicit model, the SSQCD, and we have found that in this case, by adding a new mass term for some of the quarks, the bounce action has a parametrical dependence on the relevant couplings. The RG flow of these couplings for different regimes of scales sets the desired regions of $U V$ parameter that gives a large bounce action in the $I R$. We restricted the analysis to a region of ranks in which the model is interacting but weakly coupled, and the perturbative analysis at the non supersymmetric state is applicable. It is possible to extend this example to other SCFT theories as we explained in section 4.

It would be interesting to find some dynamical mechanism to explain the hierarchy among the different relevant perturbations, that are necessary for the stability of the metastable vacua. For example in the appendix we see that in quiver gauge theories the mass of the new quarks can be generated with a stringy instanton as in $[15,16]$. The supersymmetry breaking metastable vacua that we have found in the conformal window might be used in conformally sequestered scenarios, along the lines of [17]. Another application is the study of Yukawa interactions along the lines of [18, 19]. Superconformal field theories naturally explain the suppression of the Yukawa couplings if some of the gauge singlet fields are identified with the $T_{i}=10_{i}$ and $F_{i}=\overline{5}_{i}$ generations of the SU(5) GUT group. Here we have shown that supersymmetry breaking in superconformal sectors is viable. It is in principle possible to build a supersymmetry breaking SCFT where some of the generation of the MSSM are gauge singlets, marginally interacting with the fundamentals of the SCFT group. In this case the Yukawa arising from these generations can be suppressed as in $[18,19]$. Since supersymmetry is broken one can imagine a mechanism of flavor blind mediation, like gauge mediation, to generate the soft masses for the rest of the multiplets of the MSSM. Closely related ideas has recently appeared in [10] and [20]. 


\section{Acknowledgments}

We are grateful to Kenneth Intriligator for valuable and stimulating discussions. We also thank Riccardo Argurio, Jeff Fortin, Sebastian Franco, Riccardo Rattazzi and Angel Uranga for comments. M.S. also thanks Silvia Penati for useful discussions on the manuscript.

A. A. is supported by UCSD grant DOE-FG03-97ER40546. L. G. and M. S. are supported in part by INFN, in part by MIUR under contract 2007-5ATT78-002. A. M. is a Postdoctoral researcher of FWO-Vlaanderen. A. M. is also supported in part by the Belgian Federal Science Policy Office through the Interuniversity Attraction Pole IAP VI/11 and by FWO-Vlaanderen through project G.0428.06.

\section{A The renormalization of the bounce action}

In the paper we analyzed the bounce action at the CFT exit scale. We distinguished the infrared bounce action $S_{\mathrm{B}, \mathrm{IR}}$ from $S_{\mathrm{B}, \mathrm{UV}}$, the action evaluated at the UV scale. Indeed in a supersymmetric field theory in the holomorphic basis the bounce action is obtained from the Lagrangian

$$
\mathcal{L}=Z_{\phi} \dot{\phi}^{2}+Z_{\phi}^{-1} V(\phi)
$$

and we have

$$
S_{\mathrm{B}, \mathrm{IR}}=S_{\mathrm{B}, \mathrm{UV}} Z_{\phi}^{3}
$$

Hence the bounce action undergoes non trivial renormalization. Here we show that our analysis, performed in the canonical basis, is consistent with (A.2), both for the ISS model and for the model in section 3.

The ISS bounce action in the UV is

$$
S_{\mathrm{B}, \mathrm{UV}}=\left(\frac{\mu_{\mathrm{UV}}}{\tilde{\Lambda}_{\mathrm{UV}}}\right)^{\frac{4 \tilde{b}}{N_{f}-\hat{N}}}
$$

In the IR this action is renormalized because of the wave function renormalization of the fields. In the paper we computed the action in the canonical basis and renormalization effects have been absorbed into the couplings. From (A.2) the IR renormalized action $S_{\mathrm{B}, \mathrm{IR}}$ is

$$
S_{\mathrm{B}, \mathrm{IR}}=S_{\mathrm{B}, \mathrm{UV}} Z_{N}^{3}
$$

where the wave function renormalization is

$$
Z_{M}=\left(\frac{E_{\mathrm{IR}}}{E_{\mathrm{UV}}}\right)^{-\gamma_{N}}
$$

We now compute $S_{\mathrm{B}, \mathrm{IR}}$ and show that indeed it is (A.4). The coupling $\mu_{\mathrm{IR}}$ and the scale $\tilde{\Lambda}_{\text {IR }}$ are given as functions of their UV values

$$
\mu_{\mathrm{IR}}=\mu_{\mathrm{UV}} Z_{N}^{-1 / 4}, \quad \tilde{\Lambda}_{\mathrm{IR}}=\tilde{\Lambda}_{\mathrm{UV}} \frac{E_{\mathrm{IR}}}{E_{\mathrm{UV}}}=\tilde{\Lambda}_{\mathrm{UV}} Z_{N}^{-1 / \gamma_{N}}
$$


By substiting on the l.h.s. of (A.4) we have

$$
S_{\mathrm{B}, \mathrm{IR}}=\left(\frac{\mu_{\mathrm{IR}}}{\tilde{\Lambda}_{\mathrm{IR}}}\right)^{\frac{4 \tilde{b}}{N_{f}-\tilde{N}}}=\left(\frac{\mu_{\mathrm{UV}} Z_{N}^{-1 / 4}}{\tilde{\Lambda}_{\mathrm{UV}} Z_{N}^{-1 / \gamma_{N}}}\right)^{\frac{4 \tilde{b}}{N_{f}-N}}=S_{\mathrm{B}, \mathrm{UV}} Z_{N}^{3}
$$

where the last equality is obtained by substituting $\tilde{b}=2 N_{f}-3 N_{c}$ and $\gamma_{N}=2 \tilde{b} / N_{f}$. Nevertheless the bounce action in SQCD at the CFT exit scale results RG invariant. This is because the mass scales of the theory are related by the equation of motion of $N$. The relation between these scales is proportional to the gauge coupling which is constant during the running in the conformal window. For this reason the lifetime of the metastable vacuum cannot be parametrically large in SQCD.

In the model discussed in section 3 instead the bounce action depends non trivially on the relevant deformations

$$
S_{\mathrm{B}, \mathrm{IR}}=\left(\frac{\tilde{\Lambda}_{\mathrm{IR}}}{\rho_{\mathrm{IR}}}\right)^{\frac{4 N_{f}^{(2)}}{N_{f}^{(1)}-\tilde{N}}}\left(\frac{\mu_{\mathrm{IR}}}{\tilde{\Lambda}_{\mathrm{IR}}}\right)^{\frac{12 \tilde{N}-4 N_{f}^{(1)}}{N_{f}^{(1)}-\tilde{N}}}
$$

The UV bounce action has the same expression but in term of the UV couplings and scale. The IR coupling and scale are related to the UV values as

$$
\mu_{\mathrm{IR}}=\mu_{\mathrm{UV}} Z_{N}^{-1 / 4} \quad \rho_{\mathrm{IR}}=\rho_{\mathrm{UV}} Z_{N}^{-\frac{\gamma_{p}}{\gamma_{N}}} \quad \tilde{\Lambda}_{\mathrm{IR}}=\tilde{\Lambda}_{\mathrm{UV}} Z_{N}^{-\frac{1}{\gamma_{N}}}
$$

The infrared bounce action is then

$$
S_{\mathrm{B}, \mathrm{IR}}=S_{\mathrm{B}, \mathrm{UV}} Z_{N}^{A}
$$

where

$$
A=\frac{4 N_{f}^{(2)}\left(\gamma_{p}-1\right)}{\left(N_{f}^{(1)}-\tilde{N}\right) \gamma_{N}}+\frac{\left(3 \tilde{N}-N_{f}^{(1)}\right)\left(4-\gamma_{N}\right)}{\left(N_{f}^{(1)}-\tilde{N}\right) \gamma_{N}}=3
$$

The last equality can be obtained by substituting the relations $\gamma_{\phi_{i}}=3 R\left[\phi_{i}\right]-2$, with $R[N]=2 y$ and $R[p]=(n-x+y) / n$. Hence we verified the general result (A.2) concerning the renormalization of the bounce action.

\section{B The SSQCD}

In this appendix we review the SSQCD defined in [6] and its behavior under Seiberg duality. The model is a $\mathrm{SU}\left(N_{c}\right)$ gauge theory with quarks charged under the $\mathrm{SU}\left(N_{f}^{(1)}\right) \times \mathrm{SU}\left(N_{f}^{(2)}\right)$ flavor symmetry and a singlet in the bifundamental of $\mathrm{SU}\left(N_{f}^{(2)}\right)$. The matter content is given in table 2 . The superpotential is

$$
W=S P \tilde{P}
$$

In the conformal window, $3 / 2 N_{c}<N_{f}^{(1)}+N_{f}^{2)}<3 N_{c}$ there is a Seiberg dual description, with $\mathrm{SU}\left(N_{f}^{(1)}+N_{f}^{(2)}-N_{c}\right)$ magnetic gauge group with matter content given in table 1 , 


\begin{tabular}{|c||c|c|c|}
\hline & $N_{f}^{(1)}$ & $N_{f}^{(2)}$ & $N_{c}$ \\
\hline$\tilde{Q}+Q$ & $\bar{N}_{f}^{(1)} \oplus N_{f}^{(1)}$ & 1 & $N_{c} \oplus \bar{N}_{c}$ \\
$\tilde{P}+P$ & 1 & $\bar{N}_{f}^{(2)} \oplus N_{f}^{(2)}$ & $N_{c} \oplus \bar{N}_{c}$ \\
$S$ & 1 & $\bar{N}_{f}^{(2)} \otimes N_{f}^{(2)}$ & 1 \\
\hline
\end{tabular}

Table 2. Matter content of the SSQCD.

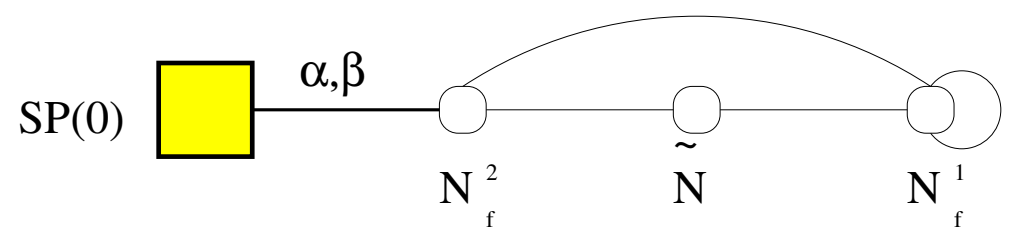

Figure 7. The Stringy instanton contribution.

where the mass term for the field $S$ and the meson $M=P \tilde{P}$ is integrated out. The dual superpotential is

$$
W=K p \tilde{q}+L \tilde{p} q+N q \tilde{q}
$$

In the conformal window these theories are dual if there are no accidental symmetry, not manifest in the UV Lagrangian, that emerges in the IR.

If some accidental symmetry arise, some gauge invariant operator, $\mathcal{O}$, in the chiral ring, violates the unitary bound and we have $R(\mathcal{O})<2 / 3$ from the a-maximization.

The marginal term in the superpotential associated to this operator becomes irrelevant and can be neglected in the IR.

In SSQCD the first operator that hits the unitary bound is $N=Q \tilde{Q}$. By using the $y_{\max }$ that we calculated in $(3.25)$ we see that the unitary bound is hit at

$$
x=\frac{1}{3}\left(2-2 n+\sqrt{1-14 n+13 n^{2}}\right)
$$

where $x=\frac{\tilde{N}}{N_{f}^{(1)}}$ and $n=\frac{N_{f}^{(2)}}{N_{f}^{(1)}}$. For higher values of $x$ the dual superpotential becomes

$$
W=K p \tilde{q}+L \tilde{p} q
$$

In the paper we have studied a region were this meson does not hit the unitary bounds, and we can trust the duality without adding new operators.

Relevant deformations. Some deformations must be added to (B.2) to recover (3.1). The linear term for $M$ can be generated in the electric gauge theory by adding a mass term for the quarks $Q$ and $\tilde{Q}$, while the mass term for the field $p$ and $\tilde{p}$ can be generated by adding a linear deformation $k^{2} S$. When we integrate out the mass term $m M S$ in the magnetic theory the fields $p$ and $\tilde{p}$ acquire a mass term proportional to $\rho=k^{2} / m$.

However a large hierarchy is required between the scale $\mu$ and the mass $\rho$ for the existence of the metastable vacua. We can impose this hierarchy at hand or find a dynamical 


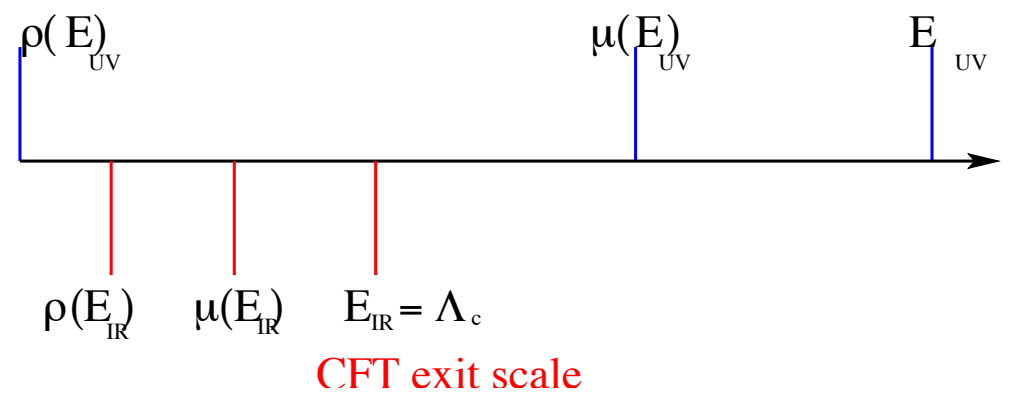

Figure 8. The evolution of the scales from the UV (above) to the IR (below).

mechanism. For example, when $N_{f}^{(2)}=1$ we can think to embed the magnetic theory in a quiver and couple the fields $p$ and $\tilde{p}$ with an $S P(0)$ node as in figure 7 .

In the instantonic action an interaction $S \sim \alpha p \tilde{p} \beta$ between the instanton moduli and the fields is present. By integrating over the instantonic zero modes we are left with the desired suppressed mass term

$$
\Delta W=\int \mathrm{d} \alpha \mathrm{d} \beta e^{S_{\text {inst }}}=\Lambda e^{-A} p \tilde{p}
$$

for the $p$ and $\tilde{p}$ quarks, where A represents the area of curve associated to the $S P(0)$ node and $\Lambda$ is associated to a string scale.

\section{RG flow and evolution of the scales}

In this appendix we summarize the evolution of the scales in the RG flow from the UV to the IR. We perturb the conformal theory by a mass term $\rho$ and by a linear term $\mu$. As we discussed in the paper a large hierarchy has to be imposed between them. At the UV scale $E_{\mathrm{UV}}$ we have

$$
\rho\left(E_{\mathrm{UV}}\right) \ll \mu\left(E_{\mathrm{UV}}\right) \ll E_{\mathrm{UV}}
$$

As we observed in the analysis of the RG flow, in the IR the mass $\rho$ grows, while the linear term reduces. Our computation is trustable if the $U V$ hierarchies translates in the IR to

$$
\rho\left(E_{\mathrm{IR}}\right) \ll \mu\left(E_{\mathrm{IR}}\right) \ll E_{\mathrm{IR}}
$$

where $E_{\mathrm{IR}}$ is the scale of the supersymmetric vacua, and it sets the CFT exit scale too. In figure 8 we give a representation of the evolution of these scales for the RG flow in this approximately conformal theory.

Open Access. This article is distributed under the terms of the Creative Commons Attribution Noncommercial License which permits any noncommercial use, distribution, and reproduction in any medium, provided the original author(s) and source are credited. 


\section{References}

[1] K.A. Intriligator, N. Seiberg and D. Shih, Dynamical SUSY breaking in meta-stable vacua, JHEP 04 (2006) 021 [hep-th/0602239] [SPIRES].

[2] K.A. Intriligator and N. Seiberg, Lectures on supersymmetry breaking, Class. Quant. Grav. 24 (2007) S741 [hep-ph/0702069] [SPIRES].

[3] N. Seiberg, Electric-magnetic duality in supersymmetric nonAbelian gauge theories, Nucl. Phys. B 435 (1995) 129 [hep-th/9411149] [SPIRES].

[4] K.A. Intriligator and N. Seiberg, Lectures on supersymmetric gauge theories and electric-magnetic duality, Nucl. Phys. Proc. Suppl. 45BC (1996) 1 [hep-th/9509066] [SPIRES].

[5] K.I. Izawa, F. Takahashi, T.T. Yanagida and K. Yonekura, Conformal supersymmetry breaking in vector-like gauge theories, Phys. Rev. D 80 (2009) 085017 [arXiv:0905.1764] [SPIRES].

[6] E. Barnes, K.A. Intriligator, B. Wecht and J. Wright, Evidence for the strongest version of the 4d a-theorem, via a-maximization along RG flows, Nucl. Phys. B 702 (2004) 131 [hep-th/0408156] [SPIRES].

[7] T.T. Yanagida and K. Yonekura, Semi-direct gauge mediation in conformal windows of vector-like gauge theories, Phys. Rev. D 81 (2010) 125017 [arXiv:1002.4093] [SPIRES].

[8] N. Arkani-Hamed and H. Murayama, Holomorphy, rescaling anomalies and exact $\beta$-functions in supersymmetric gauge theories, JHEP 06 (2000) 030 [hep-th/9707133] [SPIRES].

[9] N. Arkani-Hamed and H. Murayama, Renormalization group invariance of exact results in supersymmetric gauge theories, Phys. Rev. D 57 (1998) 6638 [hep-th/9705189] [SPIRES].

[10] D. Poland and D. Simmons-Duffin, Superconformal flavor simplified, JHEP 05 (2010) 079 [arXiv:0910.4585] [SPIRES].

[11] S. Franco and A.M. . Uranga, Dynamical SUSY breaking at meta-stable minima from D-branes at obstructed geometries, JHEP 06 (2006) 031 [hep-th/0604136] [SPIRES].

[12] A. Amariti, L. Girardello and A. Mariotti, Pseudomoduli dark matter and quiver gauge theories, JHEP 07 (2010) 072 [arXiv:0910.3615] [SPIRES].

[13] M.J. Duncan and L.G. Jensen, Exact tunneling solutions in scalar field theory, Phys. Lett. B 291 (1992) 109 [SPIRES].

[14] K.A. Intriligator and B. Wecht, The exact superconformal R-symmetry maximizes a, Nucl. Phys. B 667 (2003) 183 [hep-th/0304128] [SPIRES].

[15] R. Argurio, M. Bertolini, S. Franco and S. Kachru, Metastable vacua and D-branes at the conifold, JHEP 06 (2007) 017 [hep-th/0703236] [SPIRES].

[16] O. Aharony, S. Kachru and E. Silverstein, Simple stringy dynamical SUSY breaking, Phys. Rev. D 76 (2007) 126009 [arXiv:0708.0493] [SPIRES].

[17] M. Schmaltz and R. Sundrum, Conformal sequestering simplified, JHEP 11 (2006) 011 [hep-th/0608051] [SPIRES].

[18] A.E. Nelson and M.J. Strassler, Exact results for supersymmetric renormalization and the supersymmetric flavor problem, JHEP 07 (2002) 021 [hep-ph/0104051] [SPIRES]. 
[19] A.E. Nelson and M.J. Strassler, Suppressing flavor anarchy, JHEP 09 (2000) 030 [hep-ph/0006251] [SPIRES].

[20] O. Aharony, L. Berdichevsky, M. Berkooz, Y. Hochberg and D. Robles-Llana, Inverted sparticle hierarchies from natural particle hierarchies, Phys. Rev. D 81 (2010) 085006 [arXiv: 1001.0637] [SPIRES].

[21] H. Abe, T. Kobayashi and Y. Omura, Metastable supersymmetry breaking vacua from conformal dynamics, Phys. Rev. D 77 (2008) 065001 [arXiv:0712.2519] [SPIRES]. 